\title{
A Bargaining Solution With Level Structure
}

\author{
Xiao Yan ${ }^{1}$, Li Dengfeng ${ }^{2, *}$ \\ ${ }^{1}$ School of Economics and Management, Fuzhou University, Fuzhou, 350108, China \\ ${ }^{2}$ School of Management and Economics, University of Electronic Science and Technology of China, Chengdu, 611731, China
}

\section{ARTICLE INFO}

\section{Article History}

Received 30 Aug 2019

Accepted 22 Sep 2019

\section{Keywords}

Game theory

Level structure

Bargaining solution

Bankruptcy problem

\begin{abstract}
Presently, a conventional coalition structure can no more cover all the types of cooperative structures in practice, external cooperation between the coalitions also affects the payoff allocation between the participants. We propose a solution to solve the bargaining problem with level structure by defining for each coalition and each level. The solution concentrates on the bargaining of the coalitions with each other at each level. Furthermore, we discuss the applications of this solution to bankruptcy games with level structure. The proposed solution generalizes the bargaining solution with conventional coalition structure, which can be utilized in more cooperative types with level structures.
\end{abstract}

(C) 2019 The Authors. Published by Atlantis Press SARL. This is an open access article distributed under the CC BY-NC 4.0 license (http://creativecommons.org/licenses/by-nc/4.0/).

\section{INTRODUCTION}

The pure bargaining problem was firstly proposed for multi-players to bargain on a fair distribution cooperatively by using a practical cooperatively utility set, along with a notion to solve the problem with a series of axioms [1]. Much research has discussed the situation that bargaining occurs more frequently in coalitions than individuals. For instance, unions, firms, and governments [2-4]. Generally, the bargaining power of a coalition to another depends on their interior compositions. The most concerned issue in the cooperation progress could be the distribution of benefit between the coalitions and the members, which has been presented in Game Theory. The assumption is that the coalitions are fixed and agents can not leave it. A group bargaining model [5] was proposed for bargaining within coalition members. The solution also extends the previous research [1]. They adopt the delegation approach that bargaining is delegated to one particular member of the group so that the representing member's preferences become the group's preference. Vidal-Puga [6] takes a non-cooperative approach to describe a value in the bargaining problem with coalition structure.

Actually, in many practical situations, a coalition structure (i.e., the partition of players) is not an adequate description of the cooperative structure, and external cooperation between the coalitions themselves might also be relevant for deciding the payoff distribution between the players. In these cases, a more detailed mapping of the cooperation structure is needed. The notion of coalition structure value was generalized $[7,8]$ in a manner such that the payoff function will match with each possible "level structure" a value [9] (rather than to each other coalition structure). The term "level

*Corresponding author. Email: lidengfeng@uestc.edu.cn structure" means a sequence of coalition structures, each obtained from the previous by the unification of coalitions and each representing the various cooperation between coalitions of the previous coalition structure. Each coalition structure is called a "level." In a game with level structure, there is an ordered set of partitions, with the first level being a non-trivial partition of the partition of all singletons. Therefore, the latter games describe a situation where within any coalition any level, some players are closer to each other than to other players. Demange [10] also argues that in a hierarchical structure, teams are the relevant units in the decision-making process. A levels structure, on the other hand, organizes a set of players into a series of nested partitions. For instance, a firm might be structured in different divisions, which, in turn, might be divided in different departments, in which employees work. To take a decision, it might be that, first, all workers of each department have to reach a consensus, second, all departments of each division have to reach a consensus, and, third and last, all divisions have to reach an agreement, so that the firm as a whole takes the ultimate decision. Álvarez-Mozos [11] introduces a new way to exploit the information contained in a hierarchical structure by mapping it into a levels structure, without any loss of information in many settings and proposes three new solutions for games with hierarchical structure. van den Brink [12] provides an axiomatic characterization of the Owen-type value for games with two-level communication structure, which is a structure where the players are partitioned into a coalition structure such that there exists restricted communication between as well as within the a priori unions of the coalition structure.

The paper is organized as follows: In Section 2, we introduce the notation and some previous results. In Section 3, we present the bargaining solution with level structure and give some properties of the 
solution. In Section 4, we applied this solution to the carbon emission allocation problem combined with the bankruptcy model. In Section 5, we summarize the results of the paper.

\section{PRELIMINARIES}

There are non-overlapping coalitions $C_{1}, \ldots, C_{p}$ of players. Denote the set of all individuals by $N=\{1, \ldots, n\}$ and the coalition structure by $\mathcal{C}=\left\{C_{1}, \ldots, C_{p}\right\}$.

Definition 1. A coalition structure $\mathcal{C}$ over $N$ is a partition of the player set, i.e., $\mathcal{C}=\left\{C_{1}, \ldots, C_{p}\right\} \subset 2^{N}$ where $\cup_{C_{q} \in \mathcal{C}} C_{q}=N$ and $C_{q} \cap C_{r}=\varnothing$ whenever $q \neq r$.

Each $C_{q} \in \mathcal{C}$ is called a coalition. We denote by $c \in \mathbb{R}^{N}$ the vector whose $i$-th coordinate is given by $c_{i}=\left|C_{q}\right|$ if $i \in C_{q}$, where $\left|C_{q}\right|$ is the number of elements of $C_{q}$. Think of these coalitions as exogenously given bargaining coalitions such as firms, labor unions, households, and countries rather than voluntarily formed for the purpose of bargaining only. This is conceptually distinct from the game-theoretic term "coalition."

Definition 2. An n-person bargaining problem, or simply a bargaining problem over $N$ is a pair $(S, d)$, where the feasible set $S$ is a subset of the $n$-dimensional Euclidean space, and the disagreement (or breakdown) point $d$ is a point of $S$. The class of problems should satisfy the following conditions:

i. $\quad S$ is convex, bounded, and closed (it contains its boundary).

ii. There is at least one point of $S$ strictly dominating $d$.

iii. $(S, d)$ is $d$-comprehensive: If $x \in S$ and $x \geq y \geq d$, then $y \in S$.

iv. $S \subset \mathbb{R}_{+}^{n}$.

A solution of a bargaining problem is a function $\varphi$ that associates to every $(S, d)$ a feasible payoff vector $\varphi(S, d)$. A reasonable solution should satisfy the following five properties [1]:

Individual rationality (IR): For every $i \in N, \varphi_{i}(\mathcal{C}, S, d) \geq d_{i}$.

Pareto Efficiency (PE): There exists no feasible payoff vector $x$ such that $x_{i} \geq \varphi_{i}(\mathcal{C}, S, d)$ for every $i \in N$.

Invariance concerning Affine Transformation (IAT): Given $\gamma \in$ $\mathbb{R}_{++}^{N}$, and $\beta \in \mathbb{R}^{N}$, it holds that $\varphi(\mathcal{C}, \bar{S}, \bar{d})=\gamma \varphi(\mathcal{C}, S, d)+\beta$, where $\bar{S}=\gamma S+\beta$ and $\bar{d}=\gamma d+\beta$.

Independent of Irrelevant Alternatives (IIA): If there exists another bargaining problem $\left(\mathcal{C}, S^{\prime}, d\right)$ such that $S^{\prime}$ is a subset of $S$ and $\varphi(\mathcal{C}, S, d)$ belongs to $S^{\prime}$, then $\varphi\left(\mathcal{C}, S^{\prime}, d\right)=\varphi(\mathcal{C}, S, d)$.

Symmetry (SYM): If $\mathcal{C}=\{\{1\}, \ldots,\{n\}\}$ and $(S, d)$ is symmetric, then for any two individuals $i, j$, one has $\varphi_{i}(\mathcal{C}, S, d)=\varphi_{j}(\mathcal{C}, S, d)$.

Theorem 1. Let $\varphi(\mathcal{C}, S, d)$ be the solution of the bargaining problem $(\mathcal{C}, S, d)$, and it satisfies IR, IAT, IIA, and SYM. If there is a vector $x^{*} \in$ $S$ and $x^{*} \neq \varphi(\mathcal{C}, S, d)$, then $\exists i \in N$, it holds that $\varphi_{i}(\mathcal{C}, S, d) \geq x_{i}^{*}$.

Proof. We use proof by contradiction. Without loss of generality, we define $d=\boldsymbol{0}$. We assume that theorem 1 is false. Therefore, there must exist $x^{*} \in S$, so that $x^{*} \geq \varphi$. Given a bargaining problem $\left(S^{\prime}, \boldsymbol{0}\right)$, where
$S^{\prime}=\left\{y \in \mathbb{R}^{N} \mid y_{1}=\frac{\varphi_{1}}{x_{1}^{*}} x_{1}, y_{2}=\frac{\varphi_{2}}{x_{2}^{*}} x_{2}, \ldots, y_{n}=\frac{\varphi_{n}}{y_{n}^{*}} x_{n}, x \in S\right\}$ Obviously, because of $x^{*} \in S$, so $S^{\prime} \subset S, S^{\prime} \neq S$. According to IAT, $\left[\left(\varphi_{1} / x_{1}^{*}\right) x_{1},\left(\varphi_{2} / x_{2}^{*}\right) x_{2}, \ldots,\left(\varphi_{n} / y_{n}^{*}\right) x_{n}\right]$ is also the solution of $\left(S^{\prime}, \boldsymbol{0}\right),\left[\left(\varphi_{1} / x_{1}^{*}\right) x_{1},\left(\varphi_{2} / x_{2}^{*}\right) x_{2}, \ldots,\left(\varphi_{n} / y_{n}^{*}\right) x_{n}\right] \neq x^{*}$, this contradicts the assumption. Therefore, the solution of $(S, \boldsymbol{0})$ must be Pareto optimal.

Theorem 2. For the traditional problem, it has been shown that there exists a unique solution that satisfies IR, IAT, IIA, and SYM, called the Nash solution and that it solves the maximization problem [1]:

$$
\max _{x \in S, x>d} \prod_{i=1}^{n}\left(x_{i}-d_{i}\right)
$$

Definition 3. A bargaining problem with coalition structure is then specified as a triple $(\mathcal{C}, S, d)$ where $(S, d)$ is a bargaining problem and $\mathcal{C}$ is a coalition structure.

The traditional bargaining problem $(S, d)$ can be regarded as a particular case of the new bargaining problem where each individual forms one coalition, i.e., $\mathcal{C}=\{\{1\}, \ldots,\{n\}\}$. In order to extend Nash's result to coalition bargaining model, Chae and Heidhues [13] introduced a new property:

Representation of a Homogeneous Group (RHG): If a group $C_{j}$ is homogenous in bargaining problem $(\mathcal{C}, S, d)$ and $j^{*} \in G_{j}$, then $\varphi_{j^{*}}(\mathcal{C}, S, d)=\varphi_{j^{*}}\left(\mathcal{C}^{j}, S^{j}, d^{j}\right)$.

The RHG property says that a member of a homogeneous group receives what she would receive if she (or any other member of the coalition) became a representative member bargaining on behalf of the coalition. Intuitively, a homogeneous group can be replaced by a member to whom bargaining is delegated. RHG together with SYM implies that all coalition, rather than individuals, are treated equally in a non-discriminatory manner.

Theorem 3. There exists a solution satisfies PE, IAT, IIA, SYM, and $R H G$ if and only if it solves the maximization problem [13]:

$\max _{x \in S, x \geq d} \prod_{j=1}^{n}\left(\prod_{i \in C_{j}}\left(x_{i}-b_{i}\right)^{1 / c_{i}}\right)=\max _{x \in S, x \geq d} \prod_{i=1}^{N}\left(x_{i}-d_{i}\right)^{\frac{1}{c_{i}}}$ This solution is actually the weighted Nash solution [14], $N^{w}$, where for any $i \in N, w_{i}=\frac{1}{p c_{i}}$ with $p=|\mathcal{C}|$ and $c_{i}=\left|C_{q}\right|$ if $i \in C_{q}$.

The model provides theoretical underpinnings to the existing practice of treating a coalition of individuals as one bargainer in some applied fields of economics such as labor, financial, and international economics. We will generalize their result to our model by using the delegation approach. Conceptually, one can regard bargaining as being done simultaneously at different levels, between groups and between the members of each group at each level.

\section{BARGAINING PROBLEM WITH LEVELS STRUCTURE}

The concept of level structure was first proposed by Winter [9] in 1989 and later optimized by scholars to make a new concept more symbolically convenient. Nevertheless, these concepts are mathematically equivalent. This paper focuses on the bargaining problem with level structure. In the model, a restriction to cooperation is 
introduced as a finite sequence of partition defined on the player set, each of them being coarser than the previous one. And for each level's all coalitions, each coalition cannot communicate with each other.

Definition 4. A $k$-level structure over $N$ is a tuple $(N, \mathcal{L})$ which is a sequence of the partition of $N$ and $\mathcal{L}=\left\{\mathcal{C}_{0}, \mathcal{C}_{1}, \ldots, \mathcal{C}_{k}, \mathcal{C}_{k+1}\right\}$ such that $\mathcal{C}_{0}=\{\{i\} \mid i \in N\}, \mathcal{C}_{k+1}=\{N\}$. For each $i \in\{1,2, \ldots, k\}, \mathcal{C}_{i+1}$ is coarser than $\mathcal{C}_{i}$. That is to say, for each $i \in\{1,2, \ldots, k+1\}$ and $S \in \mathcal{C}_{i}$, there must exist $T \subseteq \mathcal{C}_{i-1}$ such that $S=\cup_{Q \in T} Q$. Each $S \in \mathcal{C}_{i}$ is called a union and $\mathcal{C}_{i}$ is called the $i$-th level of $\mathcal{L}$. $k$ is called the order of $\mathcal{L}$, i.e., the level structure with the order $k$ has $k+2$ levels.

In order not to cause confusion, we abbreviate $(N, \mathcal{L})$ to $\mathcal{L}$ later. We further denote by $\mathcal{L}^{N}$ the set of all level structures over the set $N$.

For the convenience of the following description, we denote the following notations: let $N_{r}$ be the coalitions' number in $\mathcal{C}_{r}, S_{j}^{i+1}\left(1 \leq j \leq N_{r}\right)$ be the $j$-th coalition of $r$-th level in $\mathcal{L}$, $N_{j}^{i+1}\left(0 \leq i \leq k, 1 \leq j \leq N_{i+1}\right)$ be coalitions' number containing $S_{j}^{i+1}$ in $\mathcal{C}_{i}$, correspondingly, $\left[S_{j}^{i+1}\right\rfloor$ be the coalition belonging to $S_{j}^{i+1}$ in $\mathcal{C}_{i}$.

Example 1. Take $N=\{1,2,3,4,5\}$. Then $\mathcal{L} \in \mathcal{L}^{N}$ given by $\mathcal{C}_{0}=\{\{1\},\{2\},\{3\},\{4\},\{5\}\}, \mathcal{C}_{1}=\{\{1,2\},\{3\},\{4,5\}\}, \mathcal{C}_{2}=$ $\{\{1,2\},\{3,4,5\}\}$ and $\mathcal{C}_{3}=\{\{1,2,3,4,5\}\}$ is a two-level structure of cooperative over $N$. The structure is shown in Figure 1.

Definition 5. A group bargaining problem with level structure is specified as a triple $(S, d, \mathcal{L})$, where $(S, d)$ is a bargaining problem and $\mathcal{L}$ is a level structure. By $\mathcal{B}(N)$ we represent the class of all bargaining problems with coalition structure where $N$ is the set of agents.

A solution of a bargaining problem with coalition structure is a map which assigns to every $(S, d, \mathcal{L}) \in \mathcal{B}(N)$ an element of $S$.

The coalitional bargaining problem can be regarded as a special case of the new bargaining problem with level structure when the order $k=1$. Therefore, the concept of level structure is the extension of the coalition structure.

Intuitively, the bargaining process on $(S, d, \mathcal{L})$ can be divided into $k+1$ steps. That is, first of all, distributing the payoff between $\left[\mathcal{C}_{k}\right]$, then distributing the payoff of $\left[S_{i}^{k}\right]$ which gains in the previous step between $\left\lfloor S_{i}^{k}\right\rfloor\left(1 \leq i \leq N_{k}\right)$. And so on up to the 0th level, every player will get their earnings.

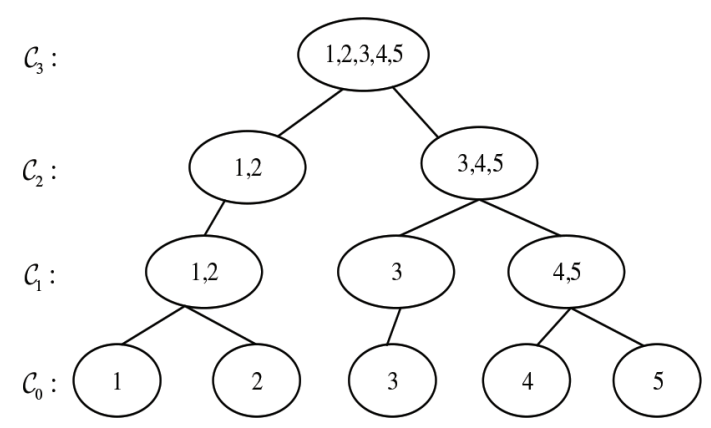

Figure 1 An example of the two-level structure.
For any bargaining problem with level structure $(S, d, \mathcal{L}) \in \mathcal{G L}$, $\mathcal{L}=\left\{\mathcal{C}_{0}, \mathcal{C}_{1}, \ldots, \mathcal{C}_{k}, \mathcal{C}_{k+1}\right\}$. The upper-right boundary $H$ of $S$ is defined as the set of points in $S$ undominated (in the weak sense of Pareto) by any point in $S$. With abuse of notation, we denote the equation of $H$ as

$$
H\left(x_{i}, x_{2}, \ldots, x_{n}\right)=0
$$

where $H$ is a function on the feasible set $S$. With no loss of generality, we assume that $H(x) \geq 0$ for all $x \in S$.

Algorithm 1: Give the initial condition: the feasible set $H\left(x_{i}, x_{2}, \ldots, x_{n}\right) \geq$ 0 , the disagreement point $d=\left\{d_{i} \mid i=1,2, \ldots, N\right\}$. The bargaining solution distribution process is as follows:

Step 1: $i \leftarrow k+1, j \leftarrow 1$, we denote $x_{\left[S_{j}^{i}\right]}=\pi$;

Step 2: If $i \geq 1$, allocate the profits $x_{\left[S_{j}^{i}\right.}$ in $\left\lfloor S_{j}^{i}\right\rfloor$,

Step 2.1: For any $\left[S_{\bar{j}}^{i-1}\right] \in\left\lfloor S_{j}^{i}\right]$, the solution is equivalent to solve linear programming as follows:

$$
\begin{aligned}
& \max \prod_{S_{j}^{i-1} \in\left[S_{j}^{i}\right]}\left(x_{\left[S_{j}^{i-1}\right]}-d_{\left[S_{j}^{i}\right]}\right) \\
& \text { s.t. }\left\{\begin{array}{c}
H\left(x_{\left[S_{j}^{i-1}\right]}\right) \geq 0 \\
x_{\left[S_{j}^{i-1}\right]} \geq d_{\left[S_{j}^{i}\right]} \\
\sum_{S_{j}^{i-1} \in\left\lfloor S_{j}^{i}\right]} x_{\left[S_{j}^{i-1}\right]} \leq x_{\left[S_{j}^{i}\right]}
\end{array}\right.
\end{aligned}
$$

Step 2.2: $j \leftarrow j+1$

Step 2.2.1: If $j>N_{i}, i \leftarrow i-1, j \leftarrow 1$, return to step 2,

Step 2.2.2: Otherwise, return to step 2;

Step 3: Otherwise, the algorithm ends. The earning of any players $i \in N\left(i=S_{i}^{0}\right)$ is $x_{i}(S, d, \mathcal{L})=x_{\left[S_{i}^{0}\right]}\left(S^{0}, d^{0}, \mathcal{L}^{0}\right)$.

Example 2. Consider the levels structure from the example 1, we assume that they divide a cake $\pi$ and $d_{i}=0$ for all $i(i=1, \cdots, 5)$. We use the Algorithm 1:

For the 3rd level, we can obtain $x_{\{1,2,3,4,5\}}=\pi$.

For the 2nd level, we need to solve the programming:

$$
\begin{aligned}
& \max \prod x_{\{1,2\}} x_{\{3,4,5\}} \\
& \text { s.t. }\left\{\begin{array}{l}
x_{\{1,2\}} \geq 0 \\
x_{\{3,4,5\}} \geq 0 \\
x_{\{1,2\}}+x_{\{3,4,5\}} \leq \pi
\end{array}\right.
\end{aligned}
$$

We can obtain $x_{\{1,2\}}=x_{\{3,4,5\}}=\frac{\pi}{2}$.

Analogously, for 1st level and 0th level, we can use the same method. And finally, we can get $x=\left(\frac{\pi}{4}, \frac{\pi}{4}, \frac{\pi}{4}, \frac{\pi}{8}, \frac{\pi}{8}\right)$.

One interesting implication of treating a coalition of individuals as one bargainer is that an individual may be worse off bargaining 
as a member of a larger coalition than as a member of a smaller coalition. In particular, an individual will be worse off bargaining as a member of a coalition than bargaining alone as can be seen easily from the above Example 1. If the levels structure is $\{\{1\},\{2\},\{3\},\{4\},\{5\}\}$, then the outcome is $\left(\frac{\pi}{5}, \frac{\pi}{5}, \frac{\pi}{5}, \frac{\pi}{5}, \frac{\pi}{5}\right)$. For player 4 and 5 , they firstly join a coalition $\{4,5\}$ and then a bigger coalition $\{3,4,5\}$. However, they get less than bargaining alone. We call this Harsanyi paradox [15]. Our interpretation for the paradox is that treating a coalition as a single bargainer reduces multiple "rights to talk" to a single right and thereby benefits the outsiders. In pure bargaining situations, where all bargainers have to agree to a reach a settlement, fewer rights to talk means reduced bargaining power. To avoid this situation, we propose the following improved algorithm.

Algorithm 2: Give the initial condition: the feasible set $H\left(x_{i}, x_{2}, \ldots, x_{n}\right) \geq$ 0 , the disagreement point $d=\left\{d_{i} \mid i=1,2, \ldots, N\right\}$. The bargaining solution distribution process is as follows:

Step 1: $i \leftarrow k+1, j \leftarrow 1$, we denote $x_{\left[S_{j}^{i}\right]}=\pi$;

Step 2: If $i \geq 1$, allocate the profits $x_{\left[S_{j}^{i}\right.}$ in $\left\lfloor S_{j}^{i}\right\rfloor$,

Step 2.1: For any $\left[S_{-}^{i-1}\right] \in\left\lfloor S_{j}^{i}\right\rfloor$, the solution is equivalent to solve linear programming as follows:

$$
\begin{gathered}
\max \prod_{S_{j}^{i-1} \in\left[S_{j}^{i}\right]}\left(x_{\left[S_{j}^{i-1}\right]}-d_{\left[S_{j}^{i}\right]}\right)^{r N_{j}^{i-1}} \\
\text { s.t. }\left\{\begin{array}{l}
H\left(x_{\left[S_{j}^{i-1}\right]}\right) \geq 0 \\
x_{\left[S_{j}^{i-1}\right]} \geq d_{\left[S_{j}^{i}\right]} \\
\sum_{S_{j}^{i-1} \in\left[S_{j}^{i}\right]} x_{\left[S_{j}^{i-1}\right]} \leq x_{\left[S_{j}^{i}\right]}
\end{array}\right.
\end{gathered}
$$

Where $r$ is a constant and $r>1 . r N_{j}^{i-1}$ represents the bargaining power of coalition $\left[S_{j}^{i-1}\right]$.

Step 2.2: $j \leftarrow j+1$

Step 2.2.1: If $j>N_{i}, i \leftarrow i-1, j \leftarrow 1$, return to step 2,

Step 2.2.2: Otherwise, return to step 2;

Step 3: Otherwise, the algorithm ends. The earning of any players $i \in N\left(i=S_{i}^{0}\right)$ is $x_{i}(S, d, \mathcal{L})=x_{\left[S_{i}^{0}\right]}\left(S^{0}, d^{0}, \mathcal{L}^{0}\right)$.

Because of the coalition's bargaining power, the members' rights actually increase with the formation of a bargaining coalition. This class of solutions can provide an independent reason why a coalition may form even in a pure bargaining situation.

\section{A NUMERICAL EXAMPLE OF CARBON EMISSION ALLOCATION COMBINED WITH THE BANKRUPTCY MODEL}

Presently, many countries are facing the pressure of energy conservation and emission reduction, so the carbon emission is in short supply. Carbon emission has become an increasing concern which can generate many environmental issues, a lot of countries has been in the suffering at present. Such an issue has a similarity with the bankruptcy problem.

Nash bargaining theory combined with bankruptcy concept was applied to allocate carbon emission by taking into account the relative utility function $f_{i}\left(x_{i}\right)$ and the minimum carbon emission allocation $\left(m_{i}\right)$ they willing to accept, i.e., disagreement allocation point $\left(m_{i}\right)$ in a convex, closed, and bounded feasibility space. The carbon emission allocation problem during bankruptcy situation can be written as $(N, E, c, f(x))$, where $N$ is the number of cities, $E$ is the total carbon emission limits for the division, and $c$ is the carbon emission claims of cities.

We assumed that the minimum carbon emission allocation to the city $\left(m_{i}\right)$ is equal to the minimal carbon emission right of each city $i$, i.e.,the amount of carbon emission which is not claimed by the other cities. $\left(m_{i}\right)$ is the minimum carbon allocation the city is willing to accept. The minimal carbon emission right of each city is defined as the following [16]:

$$
m_{i}=(E-c(N / i))=\max \{(E-c(N / i)), 0\}
$$

Given that:

$$
m(N)=\sum_{i \in N} m_{i} \leq E
$$

For a bankruptcy resource sharing problem $\left(N, E, c_{i}, f_{i}\left(x_{i}\right)\right)$, when the condition is satisfied for every resource sharing problem is called zero normalized bankruptcy problem since the minimal rights of all the claimants are zero [16]. In addition, carbon emission claims are considered unreasonable and irrational if they outstrip the available carbon emission supply. Therefore, after assigning the minimal carbon emission right each city's carbon emission demand should not be more than the available carbon emission.

$$
c_{i} \leq E-\sum_{i \in N} m_{i}
$$

The maximum possible carbon emission allocation to the cities are their respective carbon emission claims $\left(c_{i}\right)$. The utility function for cities can be defined as linear interval function ( $\mathrm{Wu}$ and $\mathrm{Xu}$ [17] as cited in Wang et al. [18]). Hence it can be formulated as follows using the carbon emission claims, disagreement carbon emission allocation and optimized carbon emission allocation of cities.

$$
f_{i}\left(x_{i}\right)=\frac{x_{i}-m_{i}}{c_{i}-m_{i}}
$$

The disagreement utility value can be obtained from the following equation:

$$
d_{i}=f_{i}\left(m_{i}\right)
$$

where:

$d_{i}$ is the disagreement utility point of each city $i$.

So, the allocation optimization problem for carbon emission allocation under a bankruptcy scenario can be written as follows:

$$
\max \prod_{i}\left(f_{i}\left(x_{i}\right)-d_{i}\right)
$$

The model is subjected to the following constraints: 
i. The utility derived from carbon emission allocated to each city should be greater than or equal to its disagreement utility point.

$$
f_{i}\left(x_{i}\right) \geq d_{i}, i=1,2, \ldots, n
$$

ii. The lower and upper carbon emission bounds for each city are its minimal carbon emission right and carbon emission demand respectively.

$$
m_{i} \leq x_{i} \leq c_{i}
$$

iii. The total allocated carbon emission should be less than or equal to the amount of water available for division.

$$
\sum_{i=1}^{n} x_{i} \leq E
$$

The model is constrained by individual rationality, efficiency while the disagreement points and claims serve as the lower and upper bounds respectively.

Algorithm 3: For any bankruptcy problem with levels structure $(E, c, f(x), \mathcal{L})$, the bargaining solution distribution process is as follows:

Step $1: i \leftarrow k+1, j \leftarrow 1$, we denote $x_{\left[S_{j}^{i}\right]}=E$;

Step 2: If $i \geq 1$, allocate the profits $x_{\left[S_{j}^{i}\right]}$ in $\left\lfloor S_{j}^{i}\right\rfloor$,

Step 2.1: For all $\left[S_{t}^{i-1}\right] \in\left[\mathcal{C}_{i-1}\right]$, calculate the minimal payoff

$m_{\left[S_{t}^{i-1}\right]}\left(\left[\mathcal{C}_{i-1}, E, c^{i-1}\right]\right)=\max \left\{(E)-\sum_{\left[\begin{array}{c}S^{i-1} \\ t^{\prime}\end{array}\right] \in\left[\mathcal{C}_{i-1}\right]\left[S_{t}^{i-1}\right]} c_{\left[\begin{array}{c}i-1 \\ S^{i-1} \\ t^{\prime}\end{array}\right]}, 0\right\}$

Step 2.2: For any $\left[S_{t}^{i-1}\right] \in\left[S_{j}^{i}\right]$, the solution is to equivalent to solve the linear programming as follows:

$$
\begin{aligned}
& \max \prod_{S_{j}^{i-1} \in\left[S_{j}^{i}\right]}\left(f\left(x_{\left[S_{j}^{i-1}\right]}\right)-d_{\left[S_{j}^{i}\right]}\right)^{r N_{j}^{i-1}} \\
& \text { s.t. }\left\{\begin{array}{l}
m_{S_{t}^{i-1}} \leq x_{\left[S_{t}^{i-1}\right]} \leq c_{S_{t}^{i-1}} \\
f\left(x_{\left[S_{t}^{i-1}\right]}\right) \geq d\left[S_{j}^{i}\right] \\
\sum_{S_{t}^{i-1} \in\left\lfloor S_{j}^{i}\right\rfloor} x_{\left[S_{t}^{i-1}\right]} \leq x_{\left[S_{j}^{i}\right]}
\end{array}\right.
\end{aligned}
$$

Step 2.3: $j \leftarrow j+1$

Step 2.3.1: If $j>N_{i}, i \leftarrow i-1, j \leftarrow 1$, return to step 2;

Step 2.3.2: Otherwise, return to step 2;

Step 3: Otherwise, the algorithm ends. The earning of any players $i \in N\left(i=S_{i}^{0}\right)$ is $x_{i}(S, d, \mathcal{L})=x_{\left[S_{i}^{0}\right]}\left(S^{0}, d^{0}, \mathcal{L}^{0}\right)$.

Example 3. Consider a country A with an administrative structure shown in Example 1. Total carbon emission limit $E=10000$,

\begin{tabular}{|c|c|c|c|c|}
\hline $\begin{array}{l}\text { Total } \\
\text { Carbon } \\
\text { Emission } \\
\text { Limit } E \\
\end{array}$ & 10000 & & & \\
\hline $\begin{array}{l}\text { Total } \\
\text { Claim } C\end{array}$ & 12000 & & & \\
\hline Cities $N$ & $\begin{array}{l}\text { The Carbon } \\
\text { Emission } \\
\text { Demand } \\
\text { (Maximum } \\
\text { Value) } c_{i}\end{array}$ & $\begin{array}{l}\text { Minimum } \\
\text { Value/ } \\
\text { Disagreement } \\
\text { Point } m_{i}\end{array}$ & $\begin{array}{l}\text { Optimization } \\
\text { Results } x_{i}\end{array}$ & $\begin{array}{l}\text { Allocation } \\
\text { Percentage } \\
p \%\end{array}$ \\
\hline City 1 & 1000 & 0 & 1000 & $100 \%$ \\
\hline City 2 & 1500 & 0 & 1500 & $100 \%$ \\
\hline City 3 & 2500 & 100 & 2235 & $89.4 \%$ \\
\hline City 4 & 3000 & 200 & 2050 & $68.3 \%$ \\
\hline City 5 & 4000 & 300 & 3215 & $86.8 \%$ \\
\hline
\end{tabular}
total claim $C=12000$, minimum value/ disagreement point $d=(0,0,100,200,300)$. According to algorithm 3 , the calculation results are shown in Table 1.
Table 1 Carbon emission allocation (Million.t) for country A.

According to the above calculation results, we can get the optimal carbon emission right of each city with an administrative structure and the allocation percentage relative to what they demand. The allocation percentage reflects the level of satisfaction they are asking for. Because the structure of each city is subject to administrative constraints, traditional bargaining model cannot explain this level structure and give the optimal solution. The level structure proposed in this paper is a generalized form of the traditional coalition structure. It can solve much more complex structural form's problems.

\section{CONCLUSIONS}

In this paper, we introduced and analyzed a bargaining model with coalitions of individuals at different levels. The model not only provides a theoretical foundation to the existing practice of considering a coalition of individuals as one bargainer in some applied fields of economics such as labor, financial, and international economics but also can potentially expand modeling tools available for these and other research areas. We extended the bargaining problem with coalition structure to level structure by defining for each player and each level and gave the calculation process of bargaining solution. Finally, we applied this model to the carbon emission allocation problem combined with the bankruptcy model.

\section{CONFLICT OF INTEREST}

No potential conflict of interest.

\section{AUTHORS' CONTRIBUTIONS}

Xiao Yan developed the model, performed the analysis, and wrote the paper. Dr. Li Dengfeng conceived the presented idea, and provided critical feedback and helped shape the research, analysis, and manuscript.

\section{ACKNOWLEDGMENTS}

This work was supported by the National Science Foundation of China (Grant Nos. 71231003). The authors would like to express their gratitude 
for the support. The authors would also like to thank the editors and anonymous reviewers for their valuable comments and suggestions.

\section{REFERENCES}

[1] J.F. Nash, The bargaining problem, Econometrica. 18 (1950), 155-162.

[2] S. Chae, P. Heidhues, Bargaining Power of a Coalition in Parallel Bargaining: Advantage of Multiple Cable System Operators, Discussion Paper FS IV 99 - 1, Wissenschaftszentrum, Berlin, 1999.

[3] R. Deneckere, C. Davidson, Incentives to form coalitions with bertrand competition, RAND J. Econ. 16 (1985), 473.

[4] B.H. Jun, Non-cooperative bargaining and union formation, Rev. Econ. Stud. 56 (1989), 59-76.

[5] S. Chae, H. Moulin, Bargaining among groups: an axiomatic viewpoint, Int. J. Game Theory. 39 (2010), 71-88.

[6] J. Vidal-Puga, A bargaining approach to the Owen value and the Nash solution with coalition structure, Econ. Theory. 25 (2005), 679-701.

[7] S. Hart, M. Kurz, Endogenous formation of coalitions, Econometrica. 51 (1983), 1047-1064.

[8] G. Owen, Values of games with a Priori Unions, in: R. Henn, O. Moeschlin (Eds.), Mathematical Economics and Game Theory, Springer, Berlin, Heidelberg, 1977, pp. 76-88.
[9] E. Winter, A value for cooperative games with levels structure of cooperation, Int. J. Game Theory. 18 (1989), 227-240.

[10] G. Demange, On group stability in hierarchies and networks, J. Polit. Econ. 112 (2004), 754-778.

[11] M. Álvarez-Mozos, R. van den Brink, G. van der Laan, O. Tejada, From hierarchies to levels: new solutions for games with hierarchical structure, Int. J. Game Theory. 46 (2017), 1089-1113.

[12] R. van den Brink, A. Khmelnitskaya, G. van der Laan, An Owentype value for games with two-level communication structure, Ann. Oper. Res. 243 (2016), 179-198.

[13] S. Chae, P. Heidhues, A group bargaining solution, Math. Soc. Sci. 48 (2004), 37-53.

[14] E. Kalai, Nonsymmetric nash solutions and replications of 2-person bargaining. J. Game Theory. 6 (1977), 129-133.

[15] J.C. Harsanyi, Rational Behaviour and Bargaining Equilibrium in Games and Social Situations, Cambridge University Press, Cambridge, UK, 1977.

[16] I.J. Curiel, M. Maschler, S.H. Tijs, Bankruptcy games, Zeitschrift für Oper. Res. 31 (1987), A143-A159.

[17] Q.L. Wu, N.R. Xu, Improved interactive methods of multiobjective decision-making based on target satisfaction degree, J. Manag. Eng. 4 (1996), 217-222.

[18] X. Wang, Y. Zhang, Y. Zeng, C. Liu, Resolving trans-jurisdictional water conflicts by the Nash bargaining method: a case study in Zhangweinan canal basin in north China, Water Resour. Manag. 27 (2013), 1235-1247. 